\title{
Oxygen Reduction Reaction Performance Tuning on Pt Nanoparticle/MWCNT Catalysts by Gd Species
}

\author{
Kentaro Ichihashi, ${ }^{\dagger}$ Satoshi Muratsugu, ${ }^{* \dagger}$ Hirosuke Matsui, ${ }^{\dagger}$ Kotaro Higashi, ${ }^{\S}$ Oki Sekizawa, \\ Tomoya Uruga, ${ }^{\S, \|}$ and Mizuki Tada* ${ }^{\dagger \dagger}$
}

$\dagger$ Department of Chemistry, Graduate School of Science, Nagoya University, Furo-cho, Chikusa, Nagoya, Aichi 464-8602, Japan.

$\S$ Innovation Research Center for Fuel Cells, The University of Electro-Communications, 1-5-1, Chofugaoka, Chofu, Tokyo 182-8585, Japan.

" Japan Synchrotron Radiation Research Institute, SPring-8, 1-1-1 Koto, Sayo, Hyogo 679-5198, Japan.

$\$$ Research Center for Materials Science/Integrated Research Consortium on Chemical Science/ Institute for Advanced Science, Nagoya University, Furo-cho, Chikusa, Nagoya, Aichi 464-8602, Japan. 
(A)

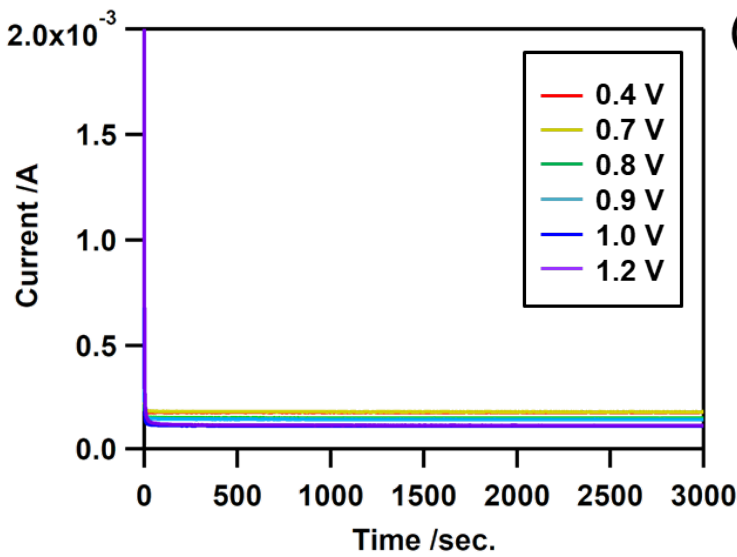

(B)

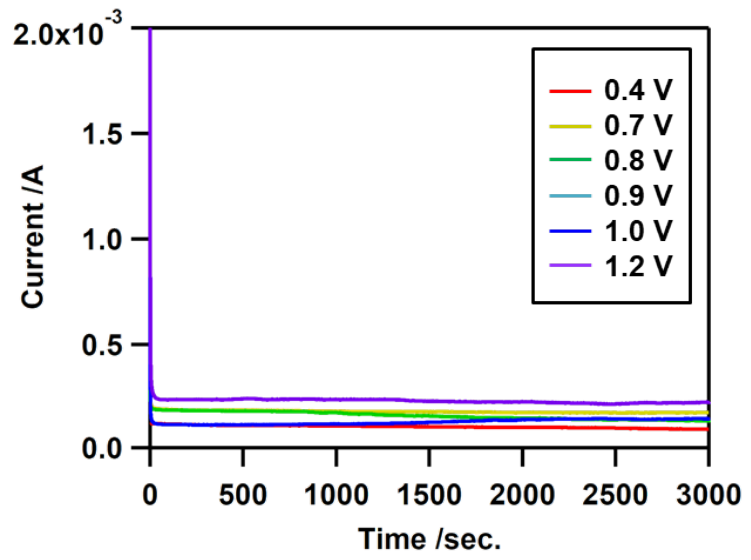

Figure S1. The current-time profiles during the applied potentials for (A) A-Pt-Gd(5/1) and (B) APt at RDE-XAFS measurements. 

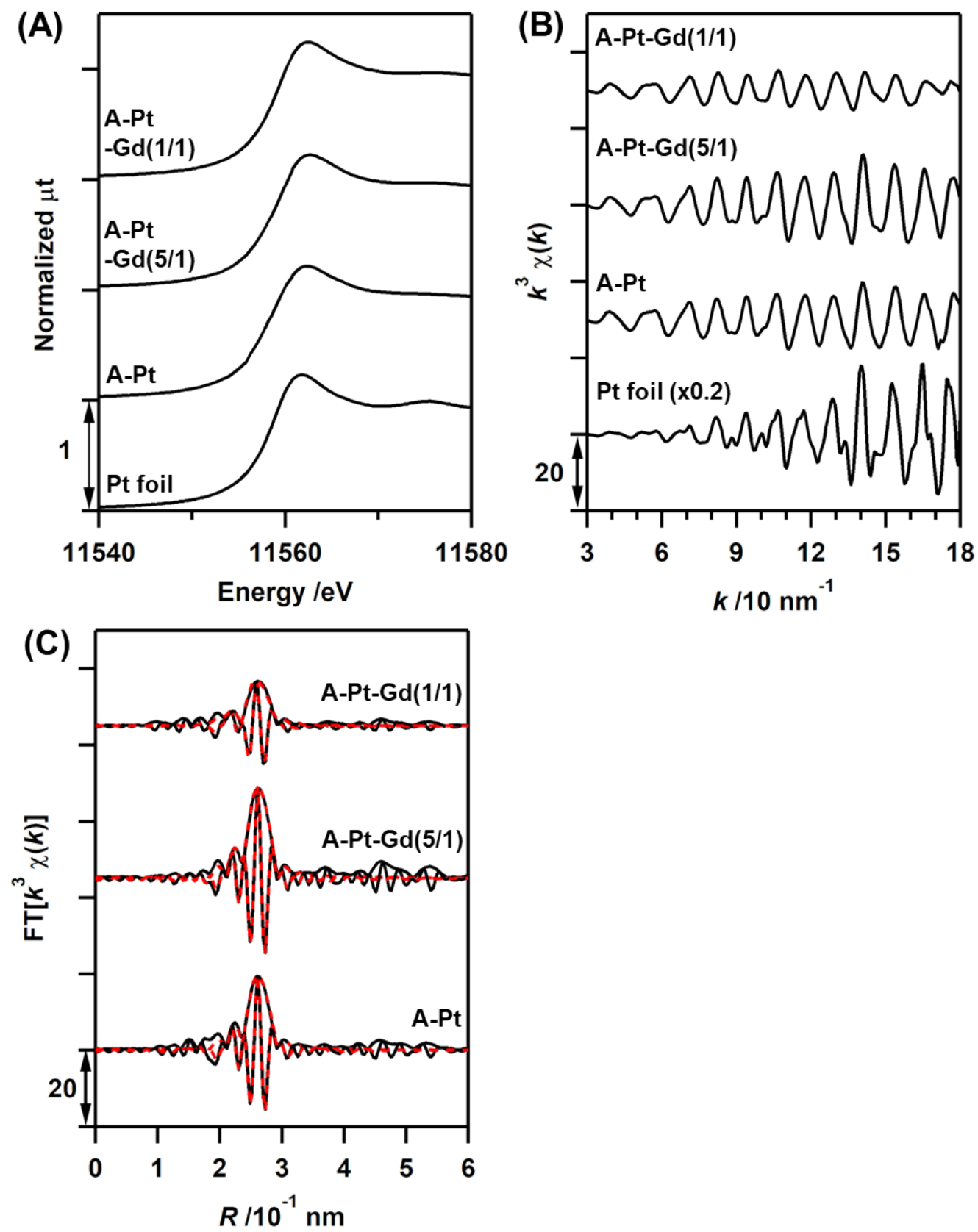

Figure S2. (A) Normalized Pt $\mathrm{L}_{\mathrm{III}}$-edge XANES spectra, (B) $k^{3}$-weighed $\mathrm{Pt} \mathrm{L}_{\mathrm{III}}$-edge EXAFS oscillations, and (C) their Fourier transforms $\left(k=30-180 \mathrm{~nm}^{-1}\right)$ for A-Pt, A-Pt-Gd(5/1), and A-PtGd(1/1) measured at $20 \mathrm{~K}$. Black solid lines in (C) show observed data, while red dashed lines show fitted data. 

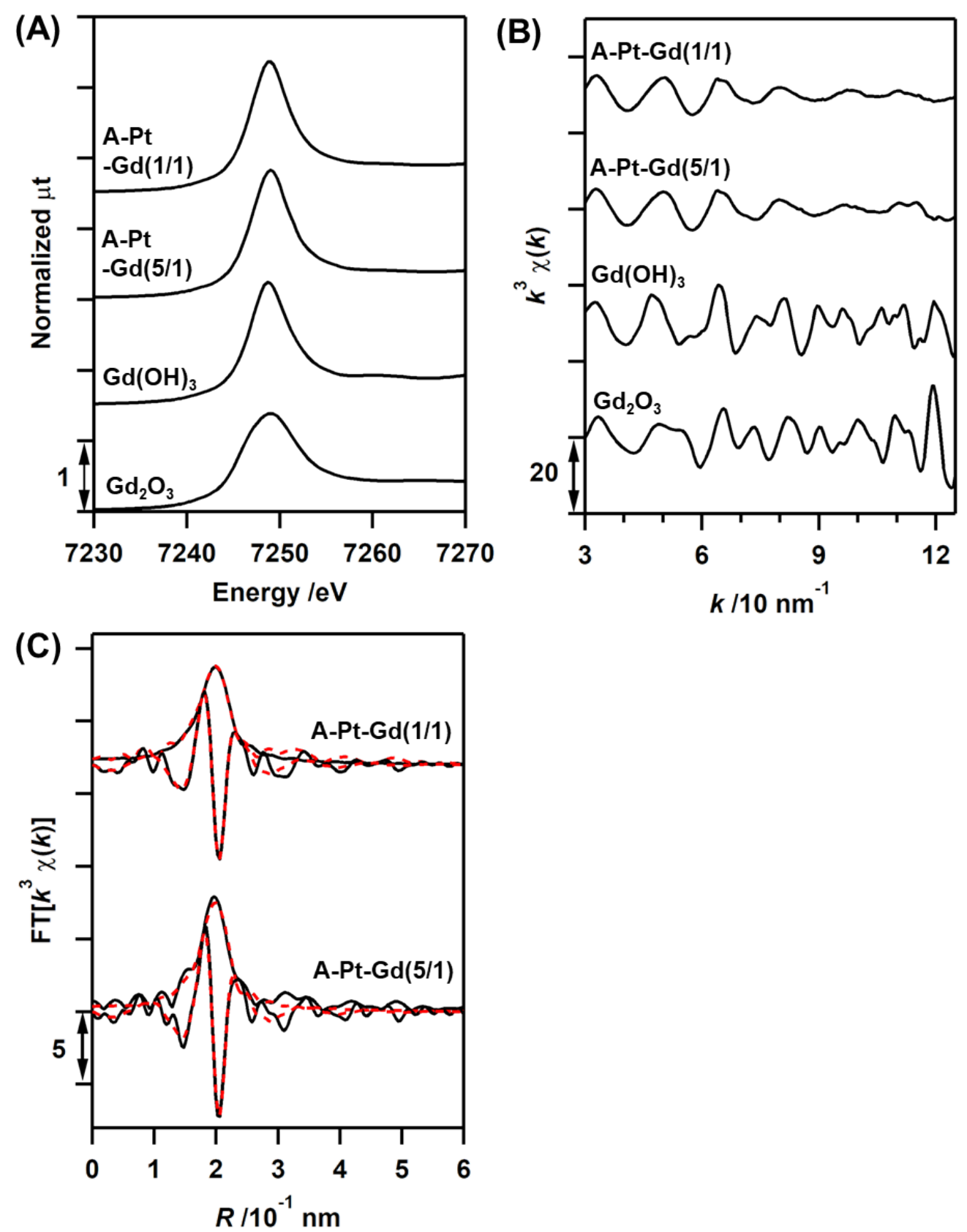

Figure S3. (A) Normalized Gd $\mathrm{L}_{\mathrm{III}}$-edge XANES spectra, (B) $k^{3}$-weighed Gd $\mathrm{L}_{\mathrm{III}}$-edge EXAFS oscillations, and (C) their Fourier transforms $\left(k=30-120 \mathrm{~nm}^{-1}\right)$ for A-Pt-Gd(5/1) and A-PtGd(1/1), and references measured at $20 \mathrm{~K}$. Black solid lines in $(\mathrm{C})$ show observed data, while red dashed lines show fitted data. 

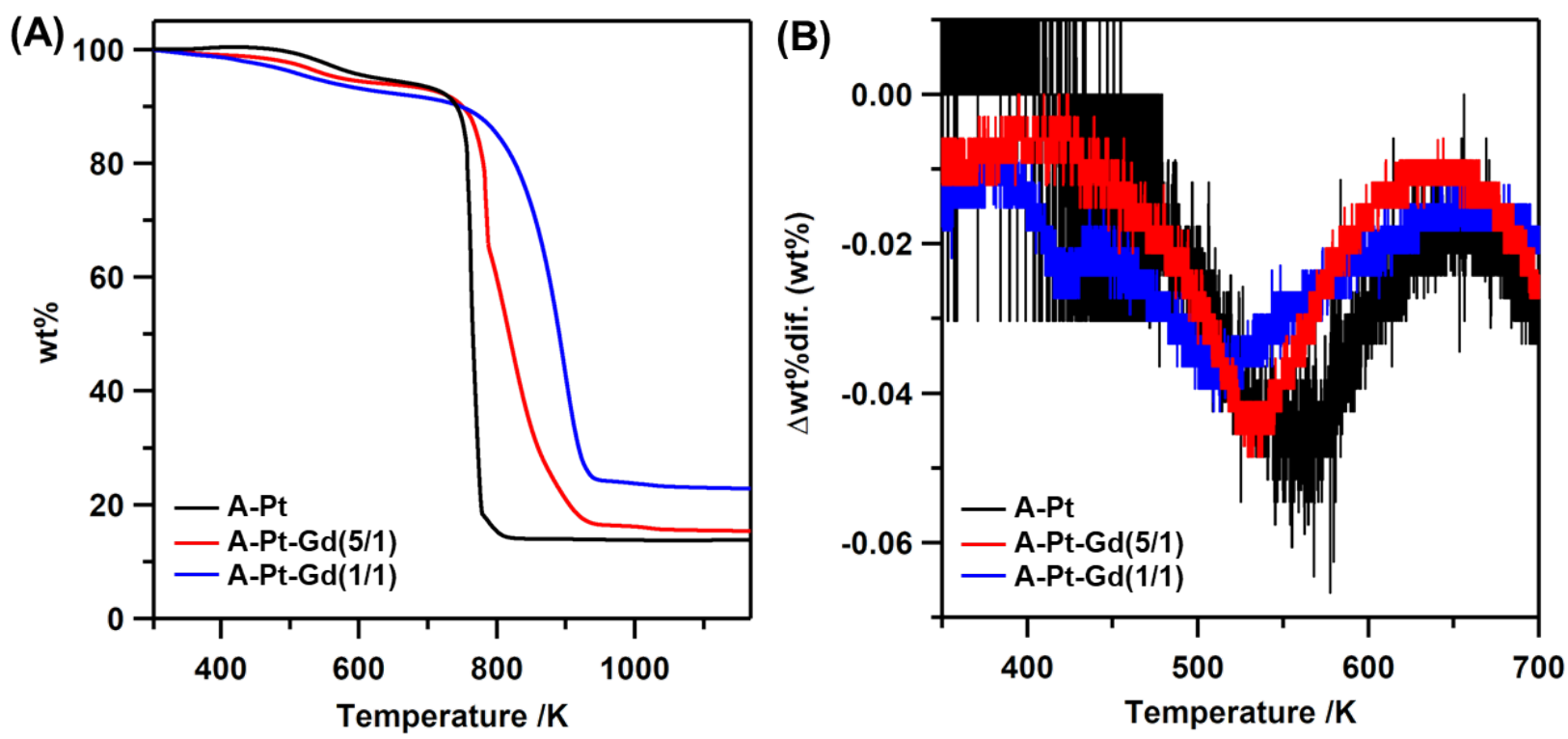

Figure S4. (A) TGA curves and (B) their $1^{\text {st }}$ differential plots of A-Pt, A-Pt-Gd(5/1), and A-PtGd(1/1). 
(A)

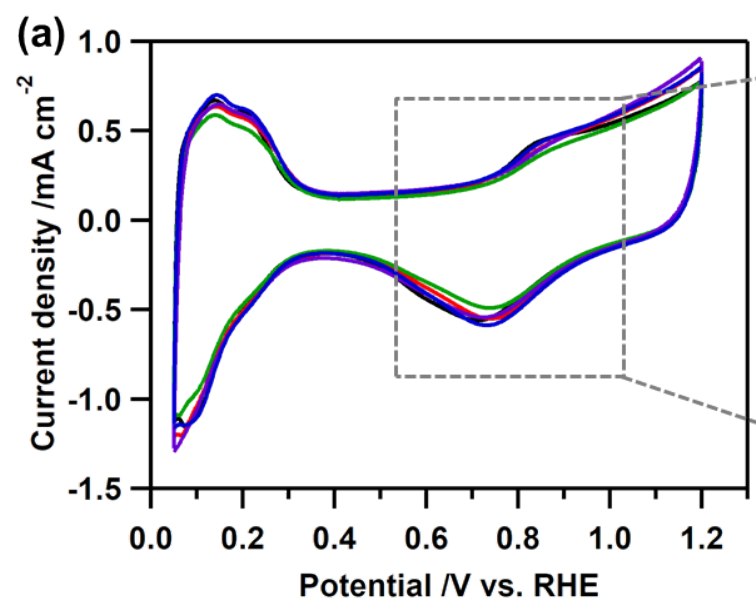

(B)

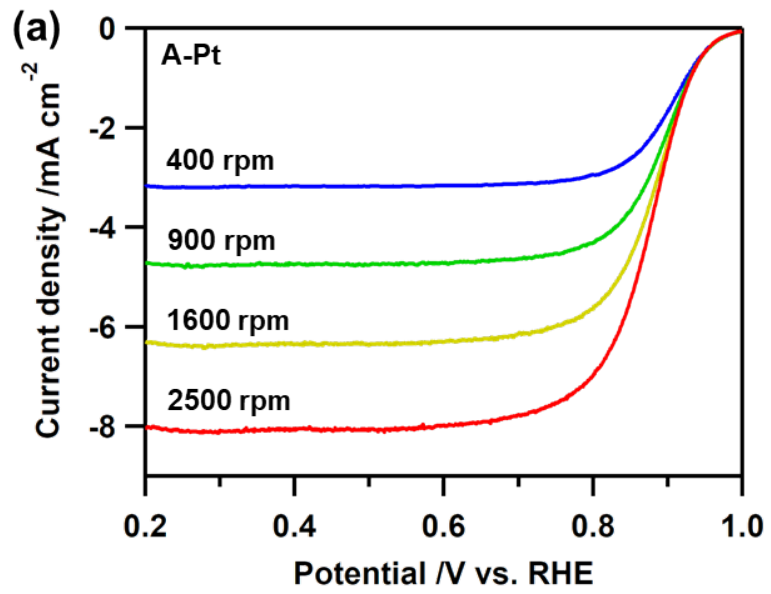

(C) (a)

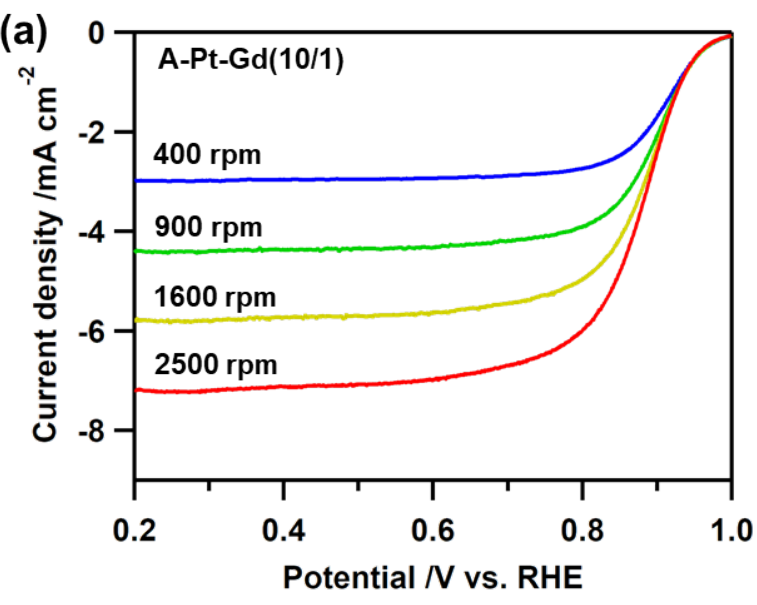

(b)

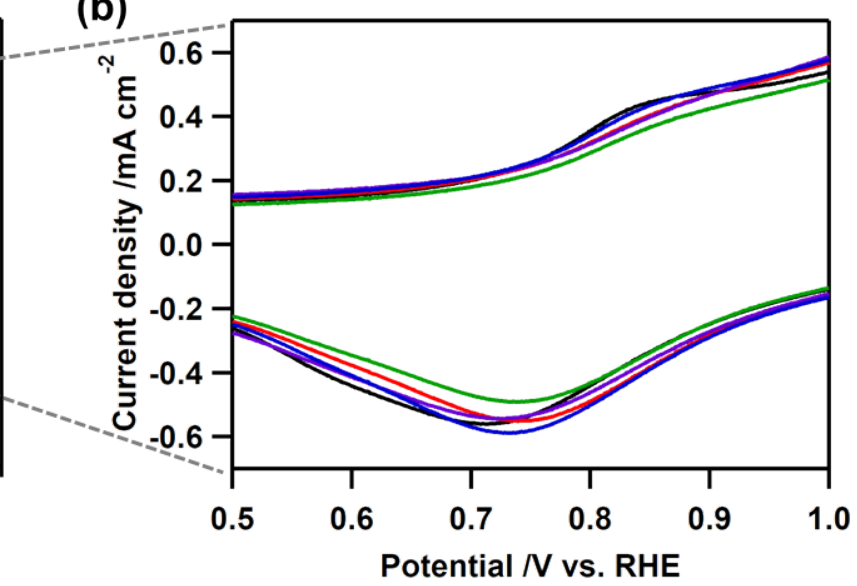

- A-Pt

- A-Pt-Gd(10/1)

- A-Pt-Gd(5/1)

- A-Pt-Gd(2/1)

- A-Pt-Gd(1/1)

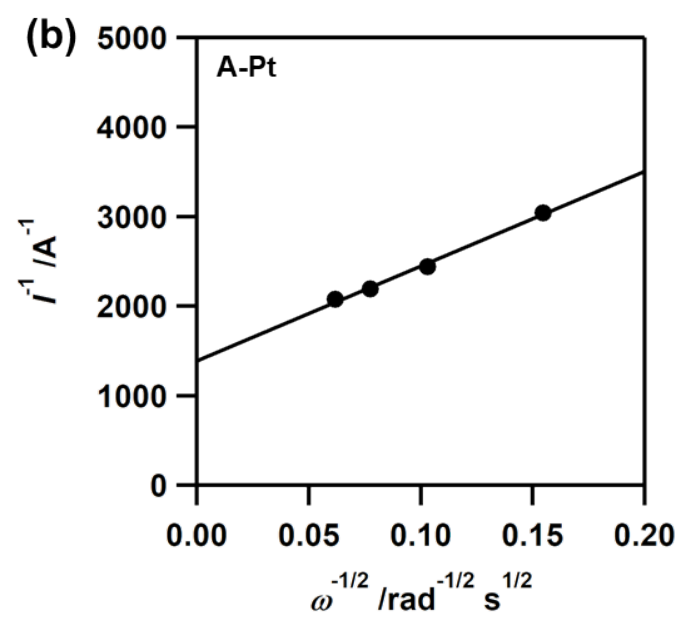

(b)

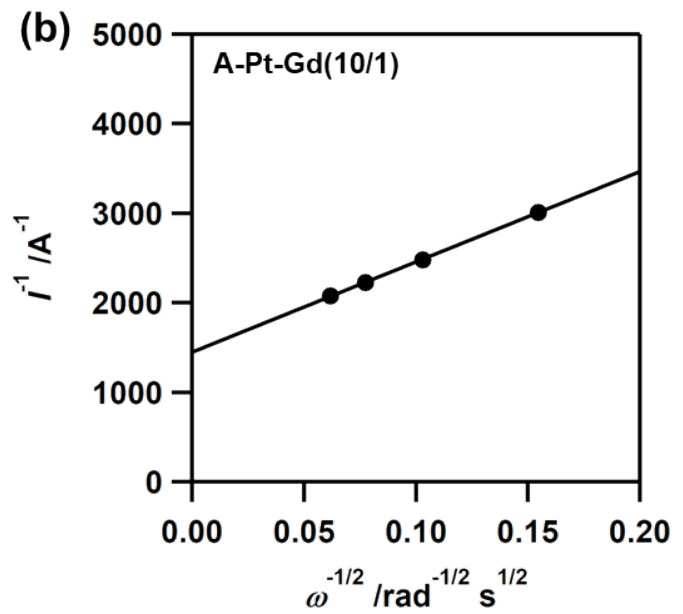

Figure S5. (A) Cyclic voltammograms of A-Pt, A-Pt-Gd(10/1), A-Pt-Gd(5/1), A-Pt-Gd(2/1), and A-Pt-Gd(1/1) recorded at a scanning rate of $50 \mathrm{mV} \mathrm{s}^{-1}$ in $\mathrm{N}_{2}$-saturated $0.1 \mathrm{M} \mathrm{HClO}_{4}$ solution. (a) Whole cyclic voltammograms, (b) enlarged view from $0.5 \mathrm{~V}$ to $1.0 \mathrm{~V}$ (vs. RHE). (B-F) (a) Linear sweep voltammograms at different rotation speeds $(400,900,1600$, and $2500 \mathrm{rpm}$ ) and (b) Koutecký-Levich plots obtained from linear sweep voltammopgrams at $0.9 \mathrm{~V}$ (vs RHE) of (B) A-Pt, (C) A-Pt-Gd(10/1), (D) A-Pt-Gd(5/1), (E) A-Pt-Gd(2/1), and (F) A-Pt-Gd(1/1) recorded at a scanning rate of $10 \mathrm{mV} \mathrm{s}^{-1}$ in $\mathrm{O}_{2}$-saturated $0.1 \mathrm{M} \mathrm{HClO}_{4}$ solution. 
(D) (a)

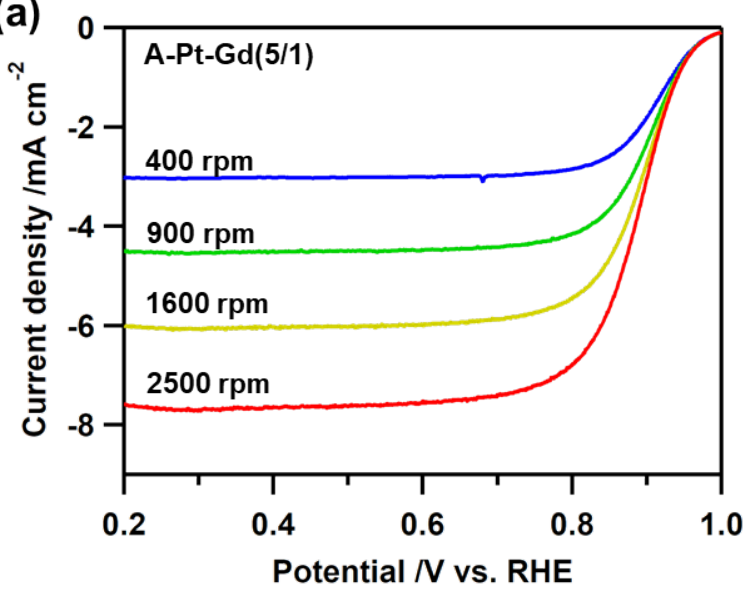

(E)

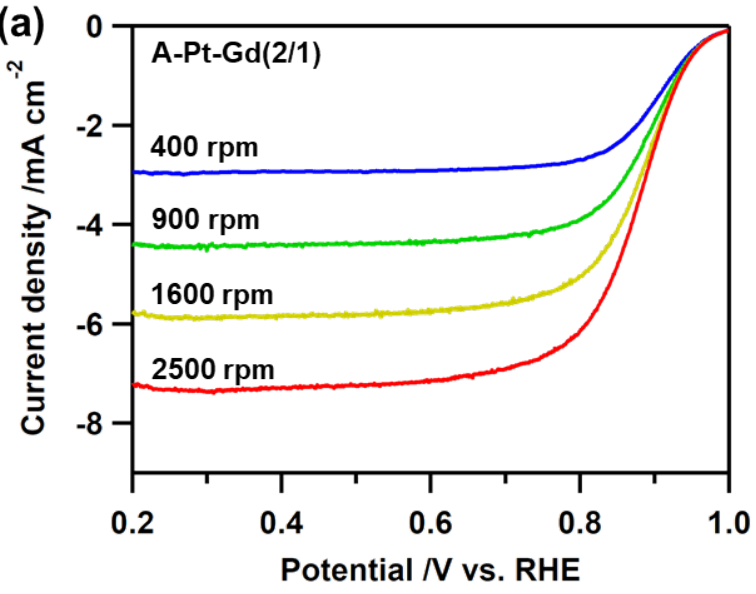

(F)

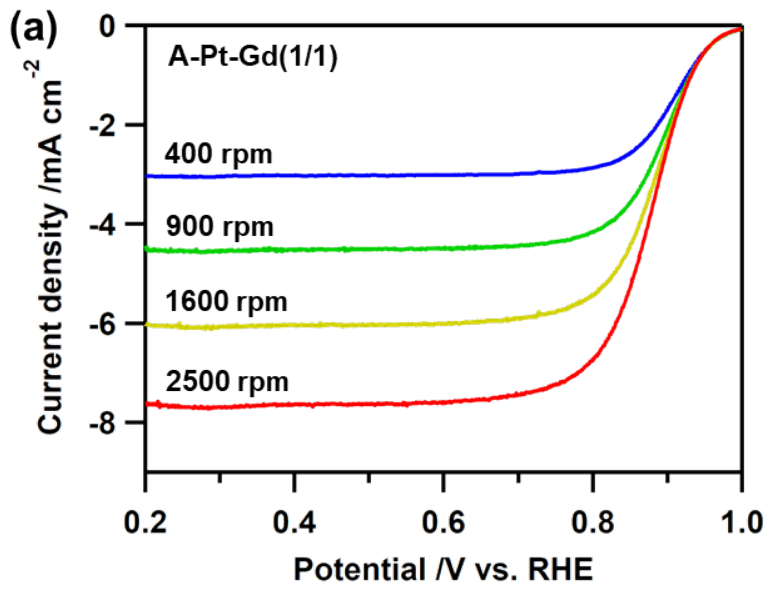

(b)

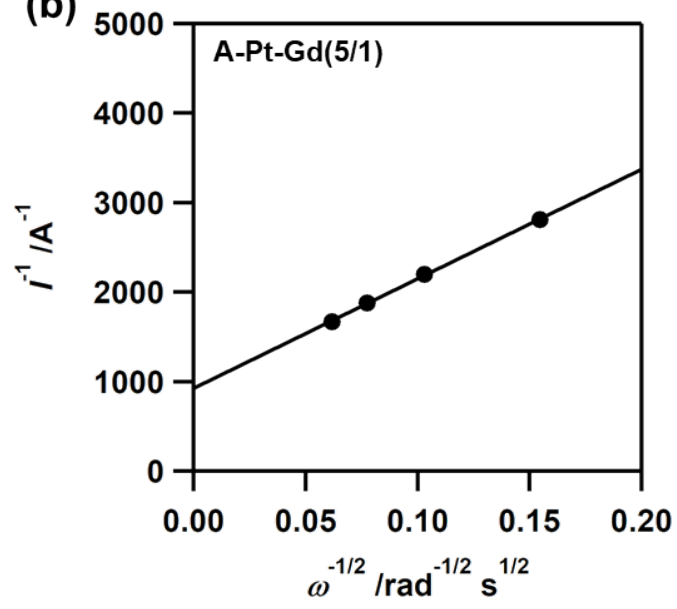

(b)

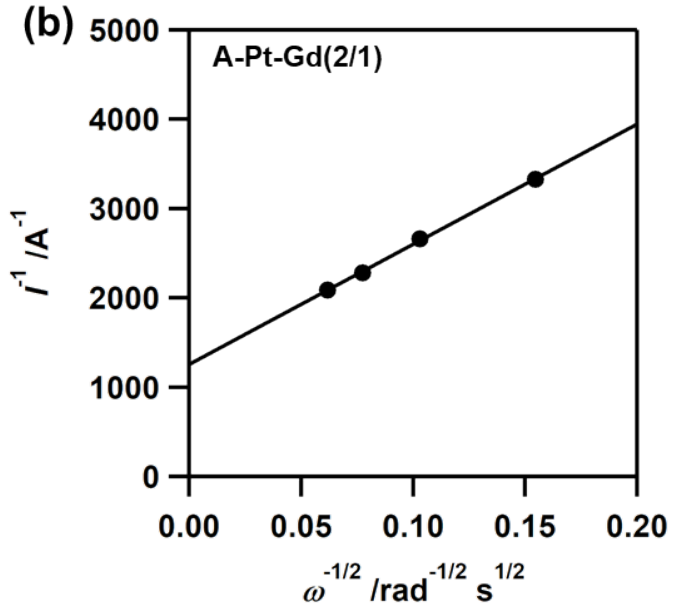

(b)

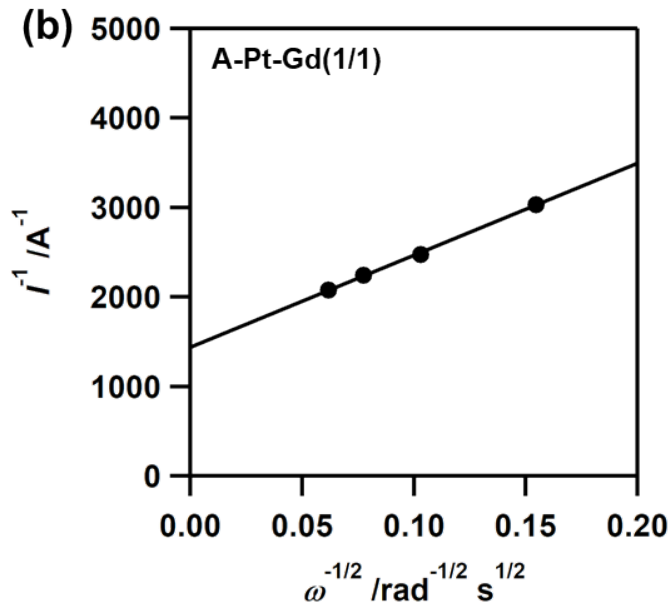

Figure S5 (Continued). (A) Cyclic voltammograms of A-Pt, A-Pt-Gd(10/1), A-Pt-Gd(5/1), A-PtGd(2/1), and A-Pt-Gd(1/1) recorded at a scanning rate of $50 \mathrm{mV} \mathrm{s}^{-1}$ in $\mathrm{N}_{2}$-saturated $0.1 \mathrm{M} \mathrm{HClO}_{4}$ solution. (a) Whole cyclic voltammograms, (b) enlarged view from $0.5 \mathrm{~V}$ to $1.0 \mathrm{~V}$ (vs. RHE). (B-F) (a) Linear sweep voltammograms at different rotation speeds (400, 900, 1600, and $2500 \mathrm{rpm})$ and (b) Koutecký-Levich plots obtained from linear sweep voltammopgrams at $0.9 \mathrm{~V}$ (vs RHE) of (B) A-Pt, (C) A-Pt-Gd(10/1), (D) A-Pt-Gd(5/1), (E) A-Pt-Gd(2/1), and (F) A-Pt-Gd(1/1) recorded at a scanning rate of $10 \mathrm{mV} \mathrm{s}^{-1}$ in $\mathrm{O}_{2}$-saturated $0.1 \mathrm{M} \mathrm{HClO}_{4}$ solution. 
(A)

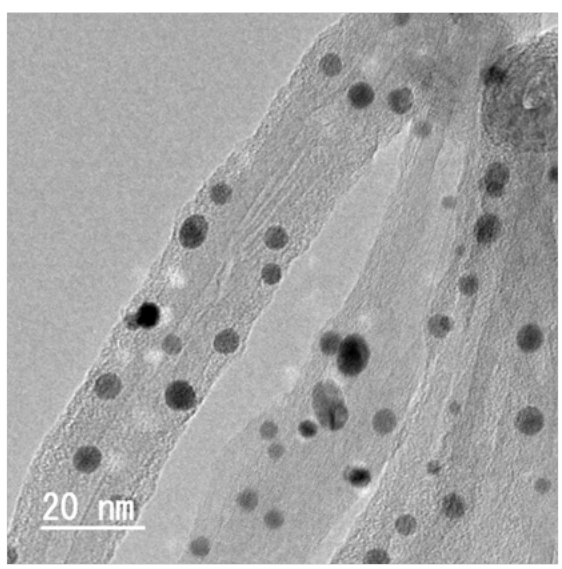

(B)

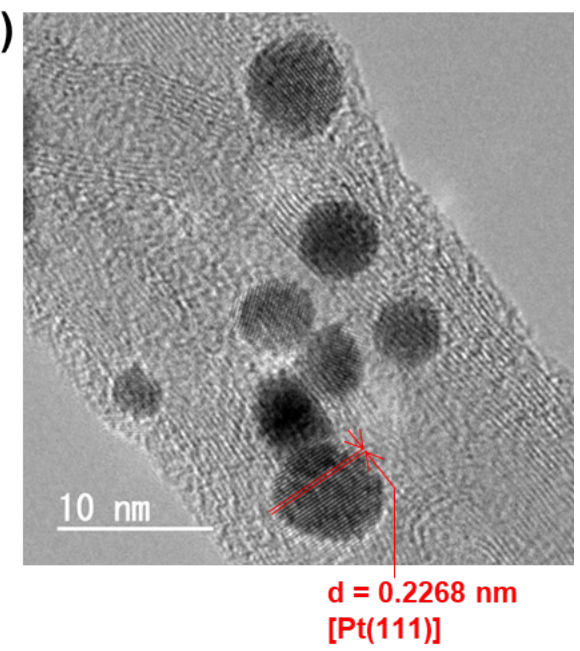

Figure S6. (A) A TEM image and (B) a magnified TEM image of A-Pt-Gd(5/1) after ADT for 30,000 cycles. 
(A)
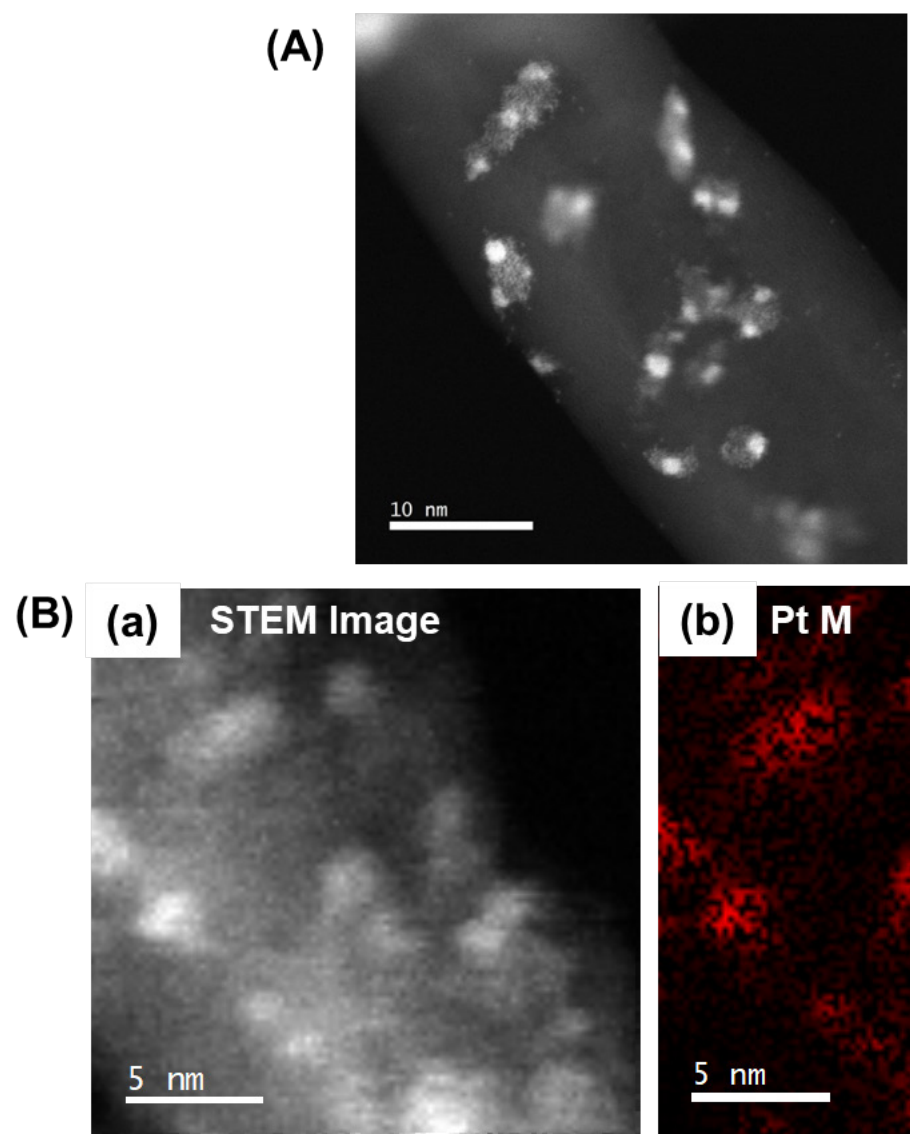

\section{(b) Pt M}
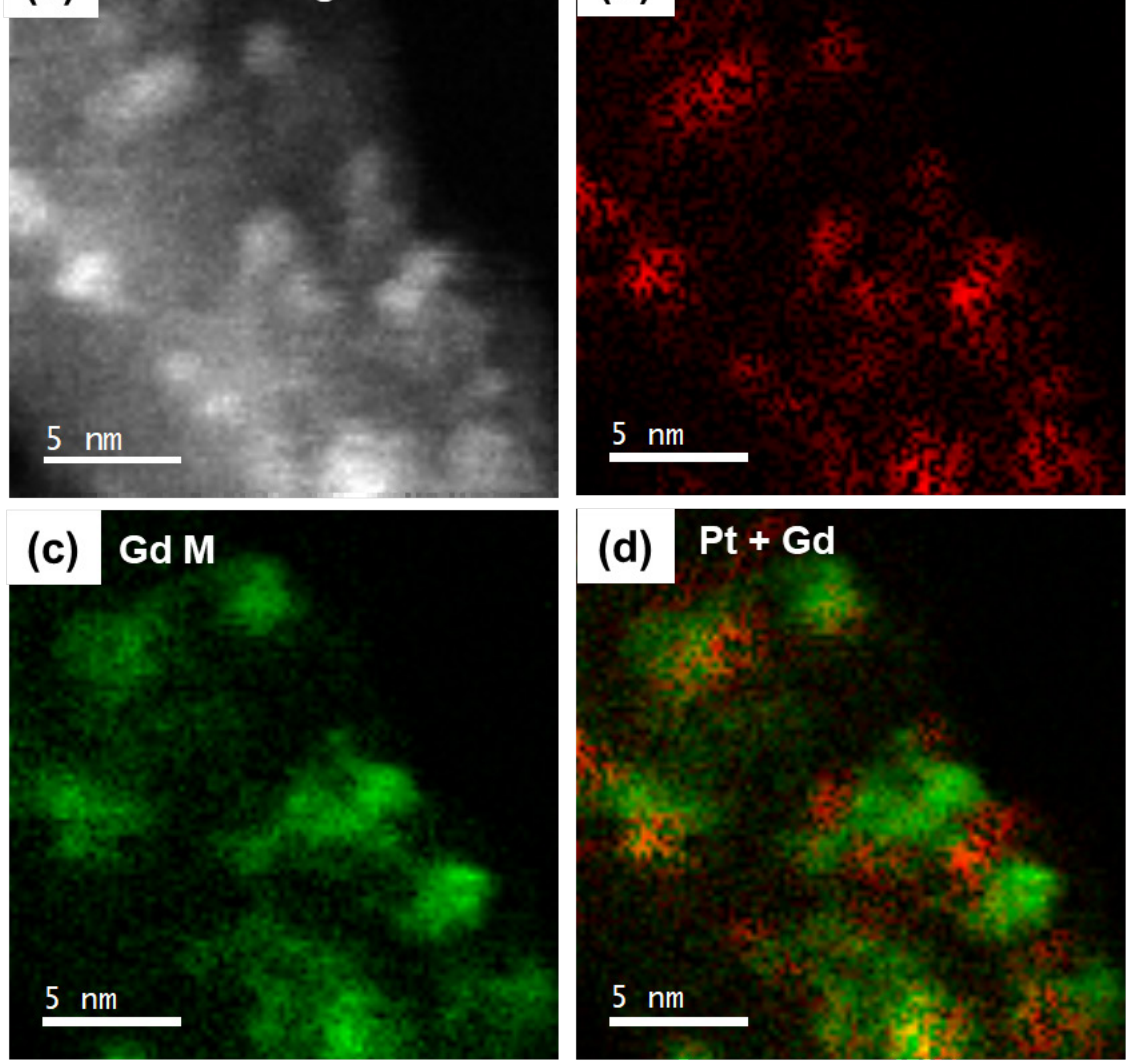

Figure S7. (A) STEM images of A-Pt-Gd(1/1). (B) STEM-EELS images of A-Pt-Gd(1/1). (a) STEM image, EELS mappings of (b) Pt ( $\mathrm{M}_{4,5}$-edge) and (c) $\mathrm{Gd}\left(\mathrm{M}_{5}\right.$-edge), and (d) overlaid mapping images (red: Pt, green: Gd). 
Table S1. Preparation Conditions and Pt/Gd Ratios for A-Pt-Gd

\begin{tabular}{ccccc}
\hline Sample & $\begin{array}{c}\text { Gd(TMHD) } \\
/ \mathrm{mg}(\mu \mathrm{mol})\end{array}$ & Acetone $/ \mathrm{mL}$ & $\begin{array}{c}\text { Pt/Gd molar ratio } \\
\text { from the complex } \\
\text { precursors used for } \\
\text { the preparation }\end{array}$ & $\begin{array}{c}\text { Pt/Gd molar } \\
\text { ratio obtained } \\
\text { from XRF after } \\
\text { the sample } \\
\text { preparation }\end{array}$ \\
\hline A-Pt-Gd(10/1) & $32.5(46.0)$ & 5 & $5 / 1$ & $10 / 1$ \\
A-Pt-Gd(5/1) & $54.3(76.8)$ & 5 & $3 / 1$ & $5 / 1$ \\
A-Pt-Gd(2/1) & $104(147)$ & 10 & $3 / 2$ & $2 / 1$ \\
A-Pt-Gd(1/1) & $164(232)$ & 12 & $1 / 1$ & $1 / 1$ \\
\hline
\end{tabular}

Table S2. Structural Parameters Determined by Curve-Fitting Analysis of the Pt $\mathrm{L}_{\mathrm{III}}$-edge EXAFS Measured at $20 \mathrm{~K}^{\mathrm{a}}$

\begin{tabular}{|c|c|c|c|c|}
\hline Shell & $\mathrm{CN}$ & $R / \mathrm{nm}$ & $\Delta E_{0}$ & $\sigma^{2} / 10^{5} \mathrm{~nm}^{2}$ \\
\hline \multicolumn{5}{|l|}{$\mathbf{A}-\mathbf{P t}^{\mathrm{b}}$} \\
\hline $\mathrm{Pt}-\mathrm{Pt}$ & $6.1 \pm 1.8$ & $0.275 \pm 0.002$ & $5 \pm 2$ & $5 \pm 1$ \\
\hline \multicolumn{5}{|c|}{ A-Pt-Gd(5/1) ${ }^{\mathrm{c}}$} \\
\hline $\mathrm{Pt}-\mathrm{Pt}$ & $6.2 \pm 0.6$ & $0.275 \pm 0.002$ & $6 \pm 2$ & $4 \pm 1$ \\
\hline \multicolumn{5}{|c|}{ A-Pt-Gd(1/1) ${ }^{d}$} \\
\hline $\mathrm{Pt}-\mathrm{Pt}$ & $5.5 \pm 0.7$ & $0.274 \pm 0.004$ & $5 \pm 2$ & $6 \pm 1$ \\
\hline
\end{tabular}


Table S3. Structural Parameters Determined by Curve-Fitting Analysis of the Gd $\mathrm{L}_{\mathrm{III}}$-edge EXAFS Measured at $20 \mathrm{~K}^{\mathrm{a}}$

\begin{tabular}{|c|c|c|c|c|c|c|c|}
\hline Shell & \multicolumn{2}{|l|}{$\mathrm{CN}$} & \multicolumn{2}{|c|}{$R / \mathrm{nm}$} & $\Delta E_{0}$ & \multicolumn{2}{|c|}{$\sigma^{2} / 10^{5} \mathrm{~nm}^{2}$} \\
\hline \multicolumn{8}{|l|}{$\mathrm{Gd}_{2} \mathrm{O}_{3}{ }^{\mathrm{b}}$} \\
\hline $\mathrm{Gd}-\mathrm{O}$ & \multicolumn{2}{|l|}{6} & \multicolumn{2}{|l|}{0.230} & --- & \multicolumn{2}{|l|}{--- } \\
\hline \multicolumn{8}{|l|}{$\mathrm{Gd}(\mathrm{OH})_{3}{ }^{\mathrm{c}}$} \\
\hline $\mathrm{Gd}-\mathrm{O}$ & \multicolumn{2}{|l|}{6} & \multicolumn{2}{|l|}{0.246} & --- & \multicolumn{2}{|l|}{---} \\
\hline $\mathrm{Gd}-\mathrm{O}$ & \multicolumn{2}{|l|}{3} & \multicolumn{2}{|l|}{0.247} & --- & \multicolumn{2}{|l|}{--- } \\
\hline \multicolumn{8}{|l|}{ A-Pt-Gd(5/1) ${ }^{\mathrm{d}}$} \\
\hline $\mathrm{Gd}-\mathrm{O}$ & \multicolumn{2}{|c|}{$7.6 \pm 1.6$} & \multicolumn{2}{|c|}{$0.239 \pm 0.009$} & $11 \pm 2$ & \multicolumn{2}{|l|}{$9 \pm 2$} \\
\hline \multicolumn{8}{|l|}{ A-Pt-Gd(1/1) ${ }^{\mathrm{e}}$} \\
\hline $\mathrm{Gd}-\mathrm{O}$ & \multicolumn{2}{|c|}{$7.8 \pm 1.1$} & \multicolumn{2}{|c|}{$0.239 \pm 0.009$} & $11 \pm 1$ & \multicolumn{2}{|l|}{$10 \pm 2$} \\
\hline \multirow{2}{*}{\multicolumn{8}{|c|}{$\begin{array}{l}{ }^{\mathrm{a}} S_{0} \text { was fitted to be } 0.965 \text { from } \mathrm{Gd}_{2} \mathrm{O}_{3} . k=30-120 \mathrm{~nm}^{-1}, R=0.12-0.30 \mathrm{~nm} .{ }^{\mathrm{b}} \text { Refere } \\
\text { Reference } 2 .{ }^{\mathrm{d}} R_{\mathrm{f}}=2.7 \% .{ }^{\mathrm{e}} R_{\mathrm{f}}=1.0 \% \text {. }\end{array}$}} \\
\hline & & & & & & & \\
\hline \multirow{2}{*}{ Sample } & \multicolumn{4}{|c|}{ Binding energy /eV } & & & \\
\hline & $\mathrm{C} 1 \mathrm{~s}$ & $\mathrm{~N}$ 1s & $\mathrm{O} 1 \mathrm{~s}$ & $\operatorname{Gd} 4 d_{5 / 2}$ & $\mathrm{Gd} 4 \mathrm{~d}_{3 / 2}$ & $\operatorname{Pt} 4 f_{7 / 2}$ & $\operatorname{Pt} 4 \mathrm{f}_{5 / 2}$ \\
\hline $\mathbf{A - P t}$ & 284.3 & 399.9 & 532.1 & --- & --- & 71.6 & 74.8 \\
\hline A-Pt-Gd(5/1) & 284.3 & 399.9 & 532.1 & 143.3 & 149.2 & 71.6 & 74.8 \\
\hline A-Pt-Gd(1/1) & 284.3 & 399.9 & 532.3 & 143.4 & 149.4 & 71.6 & 74.8 \\
\hline
\end{tabular}

${ }^{a}$ The binding energies were referenced to the $\mathrm{Au} 4 \mathrm{f}_{7 / 2}$ peak of $\mathrm{Au}$ foil $(83.96 \mathrm{eV})$.

Table S5 The Structural Parameters Determined by Curve-Fitting Analysis of the Pt 4f XPS Spectra ${ }^{\mathrm{a}}$

\begin{tabular}{llllll}
\hline \multirow{2}{*}{ Sample } & \multicolumn{3}{l}{ Binding energy /eV } & & Pt(II) ratio \\
& $\operatorname{Pt}(0) 4 \mathrm{f}_{7 / 2}$ & $\operatorname{Pt}(\mathrm{II}) 4 \mathrm{f}_{7 / 2}$ & $\operatorname{Pt}(0) 4 \mathrm{f}_{5 / 2}$ & $\operatorname{Pt}(0) 4 \mathrm{f}_{5 / 2}$ & \\
\hline A-Pt $^{\mathrm{b}}$ & 71.5 & 73.0 & 74.8 & 76.3 & $19.8 \%$ \\
A-Pt-Gd(5/1) $^{\mathrm{c}}$ & 71.5 & 73.0 & 74.8 & 76.3 & $19.7 \%$ \\
A-Pt-Gd(1/1) $^{\mathrm{d}}$ & 71.5 & 73.0 & 74.8 & 76.3 & $18.1 \%$ \\
\hline
\end{tabular}

\footnotetext{
${ }^{\text {a }}$ All spectra were fitted by four Gaussian functions with same FWHM. The peak area of $4 \mathrm{f}_{7 / 2}$ and $4 \mathrm{f}_{5 / 2}$ components were fixed at a $4: 3$ ratio, and a theoretical spin-orbit splitting of $3.3 \mathrm{eV}$ was used. ${ }^{3 \mathrm{~b}}$ $R^{2}=0.98,{ }^{\mathrm{c}} R^{2}=0.98,{ }^{\mathrm{d}} R^{2}=0.98$.
} 
[References]

(1) Inorganic Crystal Structure Database (ICSD) No. 40473. Saiki, A.; Ishizawa, N.; Mizutani, N.; Kato, M. Structural Change of C-Rare Earth Sesquioxides $\mathrm{Yb}_{2} \mathrm{O}_{3}$ and $\mathrm{Er}_{2} \mathrm{O}_{3}$ as a Function of Temperature. Yogyo Kyokaishi, 1985, 93, 649-654.

(2) ICSD No. 200093. Beall, G. W.; Milligan, W. O.; Wolcott, H. A. Structural Trends in the Lanthanide Trioxides. J. Inorg. Nucl. Chem. 1977, 39, 65-70.

(3) Lewera, A.; Timperman, L.; Roguska, A.; Alonso-Vante, N. Metal-Support Interactions between Nanosized Pt and Metal Oxides $\left(\mathrm{WO}_{3}\right.$ and $\left.\mathrm{TiO}_{2}\right)$ Studied Using X-ray Photoelectron Spectroscopy. $J$. Phys. Chem. C 2011, 115, 20153-20159. 